Miriam Ommeln: Ästhetisches Denken als Gegenargument zum Transhumanismus. Angenommen zur Veröffentlichung in: Stephan Herzberg/Heinrich Watzka (Hrsg.), Transhumanismus. Über die Grenzen technischer Selbstverbesserung. Berlin/Boston 2020, de Gruyter, S. 203-220.

\title{
Ästhetisches Denken als Gegenargument zum Transhumanismus
}

\author{
Miriam Ommeln \\ Karlsruher Institut für Technologie (KIT)
}

\begin{abstract}
Thinking Aesthetically as an Objection to Transhumanistic Arguments
The following reflections are concerned with inconsistencies and contrary expectations. They all come down into an aesthetical thinking without the mistake of trying to define itself or the Aesthetics. The appearance of beauty will be admitted likewise to technology, art or nature. But that is not the point, what matters is the specific way of thinking and gaining knowledge. After an introduction referring to transhumanistic obscurities, the paradoxical and ambiguous essential of the problem will be vivified by selected literary texts of Stefan Zweig, Heinrich von Kleist and Christoph Martin Wieland. The justification for taking into account such literature lies in the strong and lasting impression they may cause, granting possible insight into the subtle human nature. It is straightforward the very human being in all his richness who is negotiable in the mindset of Transhumanists, isn't it? Finally, the epistemological way of aesthetical thinking, ignored and left aside by the Transhumanists, will be reflected philosophically, and paradigmatically exemplified by two ideas: the figure of a dancer and a laugher.
\end{abstract}

Die nachfolgenden Betrachtungen handeln von Widersprüchlichkeiten und zuwiderlaufenden Erwartungen. Sie münden in ein ästhetisches Denken, ohne in den Fehler zu verfallen, dieses oder die Ästhetik selbst definitorisch fixieren zu wollen. Das Vorkommen von Schönheit wird gleichermaßen der Technik, wie der Kunst oder der Natur zugestanden. Doch darum geht es nicht, sondern vielmehr um eine spezifische Art und Weise des Denkens und Erkenntnisgewinns. Nach einer eher nüchtern gehaltenen Hinführung zu diesbezüglichen transhumanistischen Unklarheiten wird die Problemlage bei vorhandener Ambiguität und paradoxalen Verhältnissen anhand ausgesuchter literarischer Texte von Stefan Zweig, Heinrich von Kleist und Christoph Martin Wieland verlebendigt. Dieser Exkurs und Rückgriff auf die Literaturwissenschaft bezieht seine Rechtfertigung aus der ungeheuer starken und mehrschichtig subtilen Ausdruckskraft solcher Werke, vermöge derer ein bleibender und tiefer Einblick in die menschlichen Charaktere und ihre Wesensart gelingen kann. So ist es ja geradewegs der Mensch, der mit allen seinen Facetten und in aller Umfänglichkeit vom Transhumanismus zur Disposition gestellt wird. In einem weiteren, philosophisch reflektierenden Schritt wird der von der transhumanistischen Denkweise ignorierte und beiseitegelassene Erkenntnisweg des ästhetischen Denkens in seiner prinzipiellen Offenheit und Unausschöpflichkeit an den beiden Denkfiguren eines Tanzenden und eines Lachenden expliziert.

\section{Die Lücke}

Es ist bemerkenswert, wie leicht es oftmals ist und wie wenig argumentativer Raffinesse es bedarf, um transhumanistische Position zu vertreten oder sich von ihnen überzeugen zu lassen. Im Grunde genommen ist es ausreichend, zu betonen, dass man nur das Beste wolle, wie z.B. Krankheitsbekämpfung. Es wird zudem die Hoffnung jedes Einzelnen genährt, sich alle 
erdenklichen individuellen Wünsche erfüllen lassen zu können, dank der vorausgesetzten Omnipotenz von technologischer Machbarkeit. Jedem Einwand begegnet man mit dem Hinweis, dieser ließe sich ebenfalls technologisch je nach dem entweder einrichten oder beheben, quasi auf Knopfdruck; und die mögliche Abwertung oder Abschaffung des Menschen sei ebenfalls zu seinem eigenen Besten, da er nichts weiter als ein Teil der Natur oder ihr minderwertig beigestellt sei, und folglich und zwangsläufig nur aufgewertet werden könne und selbstredend seine Würde behalte. Zudem sollten die Angebote auf rein freiwilliger Basis und Wahl erfolgen.

Diese verkürzt-karikierte Argumentationslinie entspricht im Kern dem Ansinnen und der hohen Klaviatur auf der in der Regel gewiefte, profitable oder monopolistische Geschäftsmodelle, wie etwa eine zukünftige Wunschtraum-Mensch-Fabrik oder heutzutage das florierende und allzu oft perfide so genannte Sozialunternehmertum, oder die Durchsetzung machtpolitischer Ansprüche gespielt werden. Wissenschaftler halten es mehrheitlich für zeitgemä $\beta$, - warum auch immer -, ,solche Eingriffe nicht kategorial auszuschließen“, auf ein „Jetzt-noch-nicht“ (Grigat 2019, S. 5) zu verweisen und sich auf eine Art rückwärtsgewandte Position einer, auch philosophisch und denkerisch nachträglichen, Reparaturmöglichkeit von Technologiefolgen zu stellen. ${ }^{1}$ So gerne man an die Erfolge großer Denker anknüpft, etwa bei Albert Einstein oder Leonardo da Vinci, so gerne verdrängt man die drückenden Gewissenskonflikte des Forschers Einstein, dessen Gehirn man zudem wider seinen Willen entnahm und stahl um es Forschungszwecken zuzuführen, seine angeblich ausgeschälten Augäpfel sollten der Erinnerung dienlich sein; oder die kulturpessimistischen Prophezeiungen Leonardo da Vincis (dazu Ommeln 2016a). Der Philosoph Günter Anders hat den Begriff der „Apokalypse-

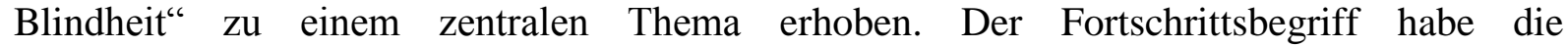
Phantasielosigkeit befördert, insbesondere die ethische Phantasie des Menschen zusehends eingeengt und beschnitten, sodass wir nun in einem, Zeitalter der Unfähigkeit zur Angst" leben (Anders 1961, S. 264). Lediglich im „Gebiete der Kunst, [...], hat man uns Freiheit gelassen“ (Anders 1961, S. 316). Galt die Jurisprudenz lange wie bei dem römischen Rechtsgelehrten Domitius Ulpianus als Wissenschaft vom Gerechten und Ungerechten, die zur Konfliktlösung bei Streitigkeiten unter Menschen beiträgt, so kommt unter dem Vorzeichen der Bio- und Informationstechnologien eine juristische Kontextausweitung und Überformung der Gesellschaft in Gange, welche durch die Juridifizierung der dinglichen Umwelt fortan das Selbstbild des Menschen von Geburt an vorprägen und modifizieren wird. Durch Patentansprüche auf Informationsgehalte kann der manipulierte Mensch leicht zum ,geliehenen' zeitlich begrenzten Leasing-Objekt unter anderen Artefakten werden (dazu Ommeln 2011). Das Bioinformationsrecht fragt daher, ,welche Persönlichkeits- oder Eigentumsrechte an extrakorporal gelagerten Körpersubstanzen oder an genetischer Information bestehen“ oder auch „ob der Eingriff in ein informationstechnisches System [...] wie ein ausgelagerter Körperteil zu behandeln ist" (Gruber 2015, Inhaltstext). Ohne auf die Risiken von geklonten und genom-editierten Organismen eigens eingehen zu wollen, sind es im hier interessierenden Fall die symbiotischen Verschmelzungen, bei denen im Hintergrund die Fäden gezogen werden und die Einfallstore für unerwünschte Fremdbestimmungen bergen. Eindringende Computer-Viren; geplante Obsoleszenz, um die Nachfrage anzukurbeln; Sinnestäuschungen aller Art zu Zwecken der Imitation oder Täuschung, wie der Stimmen, Handschriften, der Bilder, der Situationen, der Handlungen, der Mimik und Gestik bzw. von realen Bewegungsabläufen und deren dazugehörigen personalen Identitäten. Eine den Verstand

\footnotetext{
1 Ansatzweise wird vernehmbare und konzentrierte Kritik von namhaften Experten aus dem Bereich der Künstlichen Intelligenz laut, die zur Besonnenheit aufrufen, was das Entwicklungstempo bei der Umsetzung anbelangt, wie etwa in einem offenen Brief auf der Homepage von The Future of Life Institute (FLI), der im Jahre 2015 eingestellt wurde und bis heute über 8.000 unterzeichnende Personen zählt. Dazu gehören Leitfiguren wie der Physiker Stephen Hawking, Elon Musk, CEO von Tesla Motors, oder die Entwicklungschefs von Google und Microsoft, Peter Norvig und Eric Horvitz u.v.m. (FLI 2015).
} 
und die Emotionen manipulierende Künstliche Intelligenz, unbemerkt an einer GehirnComputer-Schnittstelle eingebracht, wie die am MIT Media Lab entwickelte psychopathische KI Norman oder die Nightmare Machine (Cebrian 2018), hätten wohl verheerende Konsequenzen. Dieses Exempel demonstriert, was passieren kann, wenn selbstlernende Maschinen nicht sorgfältig trainiert werden, d.h. wenn das Ausgangsmaterial vorschnell selektiert, unter Zeitdruck oder einseitig eingesetzt wird. Eine eigene, spätere Fehleranalyse wird erheblich erschwert bis unmöglich, wenn man selbst integraler Teil des Systems ist. Hinzu kommt die menschliche Eigenart, dass man selten entdeckt, was man wirklich an sich selbst ändern würde wollen, aber an dem Anderen findet man in der Regel x-Eigenschaften, die dem ermöglichten Änderungswunsch entgegenkämen. Trotz des drückenden Bewusstseins der menschlichen Unzulänglichkeit, die in der Sichtweise von Ernst Kapp zu technischen Organprojektionen mit kulturbildender Funktion führen, steht das empfindliche, beinahe mimosenhaft anmutende Selbstwertgefühl des Individuums mitsamt all seinen jeweiligen Minderwertigkeitsgefühlen in mutig verteidigender und kämpferischer Opposition zum vereinnahmenden, nivellierenden Gruppenzwang. In Abgrenzung zum Anderen, durch die durchlebte Erfahrung seiner eigenen tragischen Individuation, im Sinne Friedrich Nietzsches, vermag der Mensch nicht nur Werte zu fordern und zu setzen, sondern bedeutend wichtiger, die Umwertungen bestehender Werte zu schaffen, und zwar jedes Mal stets aufs Neue, was ein essentieller Wesenskern des Menschen sui generis ist. „Die Unangreifbarkeit der Individualität wird mit stellvertretender Bedeutung erkauft", wie Helmuth Plessner richtigerweise festhält und ausführlich anhand der gesellschaftlichen Konventionen, der Bedeutung des Takt- und Feingefühls und des Rollenverhalten darlegt (Plessner 2003, S. 84). Die Verführung sich eine tarnende Maske zuzulegen, sei es in Form von erwerbbaren Statussymbolen oder der Übernahme von Rollenverhalten und Klischees ist zum einen verständlich, jedoch vor allem deshalb überragend relevant, weil der „Mensch ein Lebewesen ist, für das nur das Überflüssige notwendig ist" (Ortega y Gasset 1949, S. 31), doch zum anderen erwächst hieraus die Gefahr des Hinterherlaufens und dem Gruppenzwang $\mathrm{zu}$ erliegen und somit die freie Entscheidungsgewalt des Einzelnen an die Masse zu überantworten. Steigert sich die Verkettungssituation durch die Einflussnahme von Genmanipulationen ${ }^{2}$ oder in den Menschen eingebrachte neuronale Chips in Form von vernetzter digitaler Künstlicher Intelligenz kann sich in obiger Situation unversehens ein Patt oder ein Schachmatt einstellen.

Nicht zu unterschätzen sind die notwendigen digitalen Wartungsarbeiten und System-Updates. Zur Abwehr von Hackerangriffen und digitalen Viren sowie zur fortschreibenden Funktionalitätsverbesserung und Variantenänderung gedacht, lauert in ihnen ein inhärentes Problem. Neben den Hardwaredefekten durch Materialermüdung sind es die nicht vermeidbaren Programmierfehler, im Mittel zwei pro tausend Zeilen geschriebenem Code, die sich im Laufe der Zeit festsetzen und ungeahnte Konsequenzen nach sich ziehen können. Da es aufgrund der zunehmenden Komplexität de facto unmöglich ist alles jedes Mal neu zu überprüfen, wird das Software Testing einfach ganz pragmatisch unter dem Aspekt von verfügbarer Zeit, Geldmittel und Ressourcen durchgeführt. Ein Set of Requirements dient als Richtschnur, das heißt, dass quasi ein Eckpunkte-Programm die Nutzungsbedingungen im jeweiligen Kontext verifiziert und validiert. Man kann sowohl als Theoretiker als auch als betroffener, verobjektierter Mensch nur Mannigfaches hoffen...

Trotz allem relativ neuartigem Denken zum Beispiel in den Schlagwörtern von Prozesshaftigkeit, Systemdenken und selbstlernenden Systemen sind wir im Grunde genommen der naheliegendsten und einfachsten Denkweise des Baukastenprinzips und der „Einschachtelung“ verhaftet geblieben, wie meines Erachtens immer noch gültig, bereits Johann Wolfgang von Goethe schrieb: „Der Begriff vom Entstehen ist uns ganz und gar

\footnotetext{
${ }^{2}$ Seltenst ist ein Gen für eine einzige Eigenschaft zuständig, von den Umwelteinflüssen ganz zu schweigen.
} 
versagt“, woraus folgt, dass „uns der atomistische Begriff nahe und bequem zur Hand ist, deshalb wir uns nicht scheuen, ihn auch auf organische Fälle anzuwenden" (Goethe 1998, S. 521). Es ist für uns beispielsweise bereits beschwerlich und schwer vorstellbar ein Denken in Oszillationen, Interferenzen, oder mit kritischen Massenpunkten, Schwellenwerten, Umschlagpunkten oder logarithmischen Entwicklungen, wie in den physikalischen Gesetzen gang und gäbe, oder nicht-binäre Relationen etc. intuitiv nachzuvollziehen. Dahingehend ist die Vorstellung einer kreativen Neuschöpfung aus dem Nichts schlichtweg undenkbar. Die Vereinfachung und Vereinseitigung aller Dinge liegt in der Natur des Menschen, weshalb er sich gewöhnlich erst um eine korrigierende Behebung bemüht, wenn die Umstände ihn regelrecht dazu zwingen. ${ }^{3}$ Die implizite Unterlegung eines feststehenden Weltbildes und Naturbegriffs, wie sie für die transhumanistische Legitimierung möglicher irreversibler Eingriffe in die Biologie des Menschen zum Tragen kommt, ist nicht nur wissenschaftlich obsolet und unredlich, sondern kann manche Überraschungen aufweisen. Ein streng naturwissenschaftliches Beispiel ist die unerklärliche Massenabnahme des Ur-Kilogramms, das als eine unumstößliche Säule des vergleichenden Messens galt und somit eine der sieben Basiseinheit im Internationalen Einheitensystem (SI) bildete, was nun zur Neudefinition auf Basis der sieben Naturkonstanten am 20. Mai 2019 führt. Doch weitergehend könnten sich auch selbst die als unverbrüchlich und konstant geltenden Naturkonstanten, wie die Feinstrukturoder Gravitationskonstante noch als zeitlich veränderlich herausstellen, wie einige physikalischen Theorien vorhersagen. Nun könnte man eventuell versucht sein einzuwenden, dass ähnlich minimale Kleinigkeiten bei den transhumanistischen Menschenversuchen keinerlei Rolle spielen würden, was allerdings zu beweisen wäre, denn generell gilt: wer die kleinen Dinge nicht ehrt, ist der großen nicht wert. Was nicht nur in der Ökonomie, sondern auch in den Technikwissenschaften gilt, weswegen man sich überhaupt erst die Mühe gab das SI-System zu reformieren und dies als „Meilenstein der Wissenschafts-, Technik- und Kulturgeschichte“ begreift (vgl. PTB 2017, S. 3). Nur für grob und gröbste sowie einseitige, nicht holistische, sondern rein behelfsmäßig praktisch ausgerichtete Zwecke taugt ergo das Wegfallenlassen von Wissensbereichen.

Ein anderes, weniger streng naturwissenschaftliches und nicht minder wichtiges Beispiel zum Naturverständnis, das jedoch tief in geisteswissenschaftliche Fragestellungen hinreicht, stammt von einem Philosophen:

„Es ist traurig, die Kunst als Ursache und die Kunst als Wirkung so verschiedenartig abschätzen zu müssen: wie ungeheuer ist sie als Ursache, wie gelähmt, wie nachklingend ist sie als Wirkung! [...]. Die Natur wirthschaftet nicht klug, ihre Ausgaben sind viel grösser als der Ertrag, den sie erzielt; [...]. Oder sollte man nicht mindestens erwarten, dass Ursache und Wirkung gleich stark wären; aber wie weit bleibt die Natur hinter dieser Erwartung zurück!““ (Nietzsche, KSA 1, S. 405-406).

In diesem andersartigen weit weniger sich selbst festlegenden Ansatz wird der ethische Selbstdruck genommen, dem jeder ausgesetzt ist, der nicht seine oder keine Grenzen kennt, und rastlos von sich selbst vor sich hergetrieben wird, bis sich ihm das Wesen des Seins und die alte Gefolgschaft im Denken und Handeln verweigern. Durch die Veränderung des bestehenden Ordnungsgefüges, das sich stets in der sinnlichen Wahrnehmung niederschlägt, und durch „die Idee des aus dem Sinnlichen gerissenen Sinnlichen“ wird die Widerständigkeit der Kunst bei Jacques Rancière nolens volens zu einem spannungsgeladenen festen Bestandteil der Politik (Rancière 2008, S. 14). Dieser Gedanke ist nicht ganz neu und reicht bis Homer zurück, wo in

\footnotetext{
${ }^{3}$ Die Geschichtswissenschaft kennt indes genügend Beispiele, wo die positive Anpassung selbst im schlimmsten Falle nicht gelang.
} 
der Ilias der Schmiedegott Hephaistos für den in die Schlacht ziehenden Achill den Schild mit unvermuteten und außergewöhnlichen Bildmotiven gänzlich ausschmückt und seine Kunst zudem mit einem schimmernden Rande betonend umzog, und damit die ,erste Sozialutopie der Literatur- und Kunstgeschichte" schuf (Tobben 2018, S. 187). Die Figur des Schmiedes in Gestalt des Hephaistos steht jedoch für wesentlich mehr als nur für gesellschaftspolitische Subversion mittels Kunst oder einem möglichen Auf- und Untergehen von Kunst und Ästhetik in Ethik und Moral. Er transzendiert durch seine Haltung und der damit verbundenen Art seiner Denkweise das Kunstschaffen in den Bereich der Ästhetik. Wobei das Technische gleichfalls je nach Situation in der erfinderischen Kunstfertigkeit miteingeschlossen ist. Dadurch schaffte er es ein homerisches Gelächter auszulösen. Die Sinnfixierung wird darin ad absurdum geführt, die Situation aufgelöst und ein entwicklungsoffener Neubeginn ermöglicht. Dieses Lachen steht jenseits von Moral und Ethik, es trifft jeden unterschiedslos. Den hohen Wert, die Besonderheit und hervorstechende Bedeutung des Kunstschaffens ersieht man auch heutzutage ganz weltlich aus der höchst komplexen Bestimmung des verfassungsrechtlichen Kunstbegriffs, dem Grundrecht auf Kunstfreiheit, dass einer wertenden Differenzierung zwischen guter und verwerflicher Kunst als willkürlich moralischem Urteilen entgegenwirkt (dazu Ommeln 2014a).

Das ästhetische Verlangen des Menschen wirkt in allem und durch alles. Es ist konstitutiv und treibend als Ursache, jedoch weder von verallgemeinernder noch von einschätzbarer Wirkung und Ausstrahlungskraft. Den ästhetischen Grund kann man leugnen, aber seinen Anspruch nicht ignorieren. Sowohl das sichtbare Fanal in den Künsten und den Kunstgattungen selbst als auch das Unsichtbare in der unerklärbaren Methodik und das Dissensfähige in der Ästhetik bedürfen meines Erachtens einer Erklärung, falls man ihre Machtfülle und Existenzberechtigung, wie etwa in den transhumanistischen Machbarkeitsbestrebungen, stillschweigend übergeht oder vernachlässigt. Denn schlagwortartig gefasst gilt: Der Wunsch nach Schönheit und Ausdrucksfähigkeit ist krisenfest. Das plötzliche Erscheinen und Offenbarwerden des Kunstwerks oder der Schönheit allgemein, wie sie sich ebenso im umwertenden Lachen zeigen kann oder einem mathematischen, eleganten' Beweis, steht für sich, in völliger Freiheit. In der Freiheit, Werte umzuwerten oder zu verkörpern. Kunst ist, einmal erkannt, nicht diskutierbar. Sie ist weder ein- noch wiederholbar. Sie ist höchstens als Fälschung kopierbar, denn die Entstehung der Kunst und des ästhetischen Denkens erfolgen zuallererst entlang einem Ariadnefaden mit einem nicht in ein vorhandenes Maß passenden Maßstab. Das durchbrechende Aufblitzen und Aufzeigen der Alogie kann selbst das für sich selbst stehende Böse in der Welt als vollgültigen Aspekt einer umwertenden Verneinung und als Gewand der Schönheit plausibel machen. ${ }^{4}$ Moral und Ethik sind hingegen in Maßstabsgittern verfangen, und damit von vornherein diskutierbar und unentscheidbar-dehnbar. Ohne Platonische Prämissen und Gedankenschlüsse übernehmen zu müssen, gilt die sinngemäße Aussage von Sokrates, dass das Gute, in unserem Fall Moral und Ethik, in der Natur des Schönen Zuflucht sucht (Philebos 64e5-6). Das Unabgeschlossene und Begrenzte von moralischen und ethischen Setzungen und Begründungen lässt sich allerdings in der weiten Eindeutigkeit des A-logischen eines sich zeigenden Schönen nicht fassen und entflieht wiederum sowohl einem quantitativ als auch qualitativ normierenden Zugriff. Von diesem Grunde her, aus Mangel an Schönem im philosophischen Sinne und ästhetischem Denken verlieren Ethik und Technikwissenschaften durch ihre je eigentümliche Einseitigkeit an anhaltender Überzeugungskraft. Sie werden zum fortgesetzten Flickwerk, da nur das Kunstwerk und das Schöne die Macht und die Wesensfülle besitzen auf einen Schlag, das heißt auf einmal ein vollendet Ganzes zu präsentieren. Der

\footnotetext{
${ }^{4}$ In Anlehnung an Goethes Faust könnte man derart weiterdenken: Der Teufel kommt nicht zweimal durch dieselbe Türe, und erst recht nicht im selben Gewande. Wohl durch die gleiche Tür, mit gleichem Gewande. Doch, das kann dann auch einmal das Gegenteil bedeuten.
} 
komplette Neuanfang ist möglich, desgleichen die komplette Zerstörung ermöglicht. Eine Wiederkehr des Gleichen wird eröffnet, durchaus auch im Sinne Nietzsches mitverstanden.

Aldous Huxley fokussiert sich in seinem beeindruckenden Roman Schöne neue Welt auf moralisierend-ethische Aspekte von angewandten Wissenschaften, und wird dadurch zwangsläufig mit der Unmöglichkeit dieses Unterfangens konfrontiert. Er selbst bemerkt sorgenvoll, wenn auch mit anderen Argumenten, die Gefahr und die Vergeblichkeit des Flickwerks und erkennt völlig zu Recht: „Und darum ist diese neue Schöne neue Welt dieselbe wie die alte" (Huxley 2007, S. 9). Seiner Dystopie stellt er folgerichtig als Motto ein Zitat des Philosophen Nikolai Berdjajew voran: „Und vielleicht beginnt ein neues Zeitalter, ein Zeitalter, in dem Intellektuelle und Gebildete Mittel und Wege erwägen werden, die Utopien zu vermeiden $[\ldots . .$. .

\section{Der Umgang mit der Lücke}

In dem unterlegten Verhältnis des Menschen zur Zeit und seiner jeweils priorisierten Sinnzuweisung und historischen Bestimmung (dazu Ommeln 2016b) liegt ein psychologischer Spannungsbogen verborgen, der sich im Umgang mit dem vermeintlichen Stillstand des Zeitgeschehens äußert: in dem überaktiven, ungeduldigen Nicht-aushalten-können und der passiveren, gelassenen Unabhängigkeit und Freiheit des ergebnisoffenen Zuwartens und des sich selbst bedeckt haltenden Aufhalten-Könnens. Entsprechend pendelt der Mensch, beispielsweise neigt er in Erwartung von smarten Technologien dazu, sich ihnen entgegenzuwerfen oder, frei nach Jaron Lanier, sich dümmer als die Maschinen zu stellen, damit sie smarter wirken; oder er verbleibt wie Einstein in der Selbsterkenntnis: „Denn das wesentliche im Dasein eines Menschen meiner Art liegt in dem, was er denkt und wie er denkt, nicht in dem, was er tut oder erleidet" (Pais 1986, S. 469). Die mehrfachen Widersprüchlichkeiten des menschlichen Wesens mitsamt dem Nichtkartierten und seiner nicht auslotbaren Tiefe werden in literarischen Darstellungen teilweise eingefangen und angedeutet. Im Folgenden sollen die erwähnten Texte, deren Kenntnisse ich voraussetze, deshalb auch genauso interpretationsoffen und differierend wie möglich bleiben.

Die Schachnovelle von Stefan Zweig berührt mit ihrem Strategiespiel ein wohl gelittenes Sujet der Künstlichen Intelligenz, wobei sich nun unter Umständen noch eine Psychopathische Intelligenz mit ans Brett setzen ließe. Der Geist wird eingespannt und aufgeteilt in eine rechtwinklige, quadratische zweifarbige Netzstruktur, quasi als 64-Bit-Variable, mit Ausnahme von kleinsten diagonalen Linienzügen, und einer spiegelbildlichen Aufstellung der beiden sich gegenüberstehenden farbigen Figurenparteien. Die beiden antretenden Meister ihres Fachs könnten in der Erzählung von Stefan Zweig nicht unterschiedlicher sein. Ein zwölfjähriger Waise eines Donauschiffers, vom Pfarrer ,aus Mitleid aufgenommen“, mit Sicherheit traumatisiert, in sich zurückgezogen und interessenlos scheinend, unbegabt in den Künsten der Zahlen und Buchstaben, jedoch dem absichernden Materiellen zugetan und gehorsam die aufgetragenen Arbeiten verrichtend, darob nur Seinesgleichen akzeptierend, wird von der breiten Masse selbst als Erwachsener noch als ungehobelter und überheblicher Bauernbursche verunglimpft, - ironischerweise nicht eingedenk dessen, wie wichtig die Bauernstruktur im Schach ist -, als „unmenschlicher Schachautomat“, dem „selbst den gerissensten Journalisten nie gelang ein brauchbares Wort herauszulocken", obwohl und gerade weil ihm jedermann grob unhöflich oder unredlich entgegentritt (Zweig 1979, S. 7, 32, 15). Sein gebildeter Gegenspieler, aus vornehmen Hause mit ,erbten Vertrauen“ in der Vermögensverwaltung tätig, zudem höflich, feinfühlig und aufgeschlossen, erleidet sein Trauma in der Isolationshaft, in der ihm „als Spieler ohne sonderliche Begabung“ ein trickreich ergaunertes Schachrepetitorium in die Hände fällt, worüber der Feingeist höchst enttäuscht und erzürnt ist, da sich dieser „Nonsens“ nicht als „Goethe oder Homer“ erweist, wobei sich später „die rein graphischen Diagramme 
immerhin als eine Sprache enträtseln“ lassen, die er sodann mit „künstlerischem und lustvollen Verständnis“ in der ,persönlichen Note jedes einzelnen“ und ,seiner individuellen Führung“ genießt (Zweig 1979, S. 48, 46, 70, 69, 70, 73). Und die Masse der mehr oder weniger unkundigen Leute, was will und tut sie? Am liebsten mal wieder fremde „Gehirne sezieren“ aus Mangel an Vorstellungskraft, die ,räumliche“ Nähe ausnutzen und sich ihnen ,zu nähern“, dafür sich „die absurdesten Listen ausdenken“ und ihnen wie ein professioneller „Jäger“ Fallen zu stellen, um in irgendeiner Weise partizipieren zu können, sei es aus Neugierde, um sie ,gedemütigt" zu sehen oder die höchsteigene Wichtigkeit und Sensationsschau, vielleicht sogar in Form eines „Rekordes“, der um die Welt geht, einbringen zu können, und so wird in „wilder, ehrgeiziger Kampflust [...] keinesfalls auf das Schauspiel eines neuerlichen Kampfes verzichtet" (Zweig 1979, vgl. S. 21-23, 43-44). Es menschelt fast so schön, wie es Christoph Martin Wieland ausführlich in Der Prozess um des Esels Schatten vorführt.

Was den ehemaligen Isolationshäftling ,,interessiert und intrigriert“ ist alleine die Frage, ob es sich bei ihm um „Traumschach, ein Fieberschach“ gehandelt hatte und ob er ,sich damals vor oder jenseits der gefährlichen Klippe befand“, wofür er „um die Stellung der Partie zu überblicken, sie erst zurückmutieren mußte aus der abstrakten Ziffernwelt in die der bewegten Steine" (Zweig 1979, vgl. S. 92-93). Als ebenbürtig sich erkennend und anerkennend, wird denn auch im Spiel „,der geistige Habitus im Gegensatz der beiden Partner immer mehr körperlich plastisch“, wie auch die Schachfiguren sich immer mehr zu einem „Ornament ineinander verflochten“ und sich die „einzelnen Figuren wie Hebel verschoben“, bis die Partie um „einen hölzernen König auf einem hölzernen Brett“ eskaliert, da Vorgenannter in beleidigender und ausfallender Weise persönlich wird (Zweig 1979, S. 95-97, 22). „Mit einemmal stand etwas Neues zwischen den beiden Spielern, [...], ein leidenschaftlicher Haß. Es waren nicht zwei Partner mehr, die ihr Können spielhaft aneinander proben wollten, [...]“ (Zweig 1979, S. 102-103).

Der Waise eines Donauschiffers hat sich jedoch nie einer Verletzung der regulierten Etikette im Schachspiel zuschulden kommen lassen, selbst das provokativ wirkende Hinhalten und das kafkaesk empfundene Warten liegen innerhalb der Schachregeln. Vielmehr kann das Pausieren ebenso gut als notwendig erkannte Hilfestellung, um sich wieder fangen und die Klippen umschiffen $\mathrm{zu}$ können, und zugleich als fortgesetzte Erprobung des (möglichen) Gleichgesinnten verstanden werden, welcher so misslich den ästhetischen Rahmen verwarf. In den Augen der unkundigen Menge mag es sich um moralisch-ethische Probleme handeln, doch um das Gewinnen geht es lediglich unter ferner liefen. Was weiß der Unkundige schon von jener Kunst? Was ahnt er schon von jenem Kampf? Er vermag wohl noch weniger wahrzunehmen als die gelehrten Schüler des Malers Frenhofer in Le Chef-d'œuvre inconnu (Das ungekannte Meisterwerk) von Honoré de Balzac.

Der zwölfjährige Schiffersohn, seinem Vater bei der Arbeit zur Hand gehend, wie damals üblich, der beim „Rechnen die Finger zu Hilfe nehmen muss“ und immer alles „handgreiflich vor sich haben“ und mit Hilfe eines ,zusammenlegbaren Taschenschachs [...] sich die Stellung optisch vor Augen führen“ muss aus „Mangel an imaginativer Kraft“ (Zweig 1979, vgl. S. 7, 13-14), versteht und beherrscht korrespondierend dazu einfache, aber effektive Fertigkeiten, wie das Stecken und Lösen von Schifferknoten, was ein schrittweises, d.h. unmerklich stockendes, raumorientiertes Vorgehen verlangt, oder die Navigation, wie etwa die manuelle Tiefenmessung, bei der das Handlot schritt- bzw. armlängenweise eingeholt wird und die ebenfalls unbekannten und auch zeitlich wechselnden Bodenproben in der jeweiligen Tiefe flächenabschnittsweise begrenzt bestimmt werden. Die Bedeutung von sich stets verändernden Begrenzungen und Warte-Intervallen ist ihm also äußerst bewusst. Desgleichen ist die Fähigkeit zur Vorstellung von fließenden und starren plastischen Dimensionalitäten ausgeprägt vorhanden. Im Grunde genommen ist dem Schifferjungen ein tiefverwurzeltes Rhythmusgefühl verinnerlicht, das im weiteren Sinne dem Wunsch nach „Gedichten“ bzw. Versmaßen des ehemaligen Isolationshäftlings entgegenkommt (Zweig 1979, vgl. S. 69). In seiner konkreten 
plastischen Überformung entstehen Tanzschritte. Dazu gehörten durchaus auch solche metrischen Tanzschritte, wie sie in bester Homerischer Manier die Schiffer und Seefahrer dem mantischen und pythischen Apollon folgend, vollführten. Das speziell aus dem mimetischen Tanz heraus sich entwickelnde eigenständige Pantomimenspiel mit nur einem ,Tänzer', der in stummer Gebärde sämtliche auftretende Rollenverkörperungen selbst darstellt, entspräche der besonderen Situation des ehemaligen Isolationshäftlings, der schildert: ,gegen sich selbst spielen zu wollen, bedeutet also im Schach eine solche Paradoxie, wie über den eigenen Schatten zu springen“(Zweig 1979, S. 76). Jedoch, wie er selbst zu Recht zugeben muss: „Aber selbst diese Selbstzerteilung war noch nicht das Gefährlichste an meinem abstrusen Experiment“ und beileibe ist es auch nicht das „grauenhafte Nichts um ihn“" herum, das ihn nötigen würde auf „Überlegungspausen“ zu verzichten oder die Möglichkeiten körperlicher Begrenzungen und zu selbstgewählten ritualisierenden Distanzierungen zu ignorieren (Zweig 1979, S. 79, 77, 78). Der Stein der Erprobung enthält die Frage, ob man überhaupt oder ab wann man, sich über sich selbst derart ärgert, dass man sich selbst, und andere, bekämpft und zerfleischt. Eine Revanche wäre ja möglich und einem neuen Spiel stünde prinzipiell nichts entgegen. Der springende Punkt ist der, dass man genau in dem Moment den ästhetischen Denkrahmen verlässt, indem die moralisch-ethische Komponente zum Tragen kommt und man sich ihr einseitig überlässt. Allgemeiner formuliert, wenn man sich nur all jenen Antworten verschreibt, die Zwecke und Ziele, Sinn- und Kausalitätsverknüpfungen beinhalten, und anderslautende Antworten oder entsprechende vorhergehende Fragestellungen aussortiert wurden. Das Charakteristische des ästhetischen Denkens, nicht nur des Schachspiels, ist: „ein Denken, das zu nichts führt, eine Mathematik, die nichts errechnet, eine Kunst ohne Werke, eine Architektur ohne Substanz und nichtsdestominder erwiesenermaßen dauerhafter als alle Bücher und Werke“ (Zweig 1979, S. 20). Das Distanzbewahren-können in jeglicher Hinsicht wird zur Nagelprobe, um nicht irgendwann unversehens feststellen zu müssen: „Alles steht ganz falsch auf diesem Brett ..." (Zweig 1979, S. 107). Denn dann würde man während des Handlungs- und Erkenntnisvorgangs Narkissos gleichen, der auf der Wasseroberfläche sein Spiegelbild erblickt und sich ihm verzweifelt und vergeblich $\mathrm{zu}$ nähern versucht. Selbstverständlich gelten diese Feststellungen für Kollektive gleichermaßen wie für Individuen. Die nur am Rande davon berührte Menge, die dieses seltsame Gebaren nicht wirklich nachvollziehend und verstehend verfolgt, weder von der ästhetischen Perspektive her noch von der nervösen Bewältigungsanstrengung einer sinnlosen Sisyphusaufgabe, verbleibt unaufhörlich auf ein Gutes hoffend, und dennoch ,mit dem ungewissen Gefühl, mit knapper Not etwas Unbehaglichem und Gefährlichem entgangen zu sein“" (Zweig 1979, S. 109).

In der unersättlichen Gier einen Anderen oder etwas Anderes zu vereinnahmen und zu übervorteilen, in der Gefangenschaft des Spiegels durch die Strahlen des Abbildes eingeengt und zudem mit Nietzsche durch das für ihn unerträglich hässlich erscheinende Bild genötigt sich abzuwenden, hat der Mensch nicht nur den ästhetischen Rahmen verlassen, sondern in der Regel auch die ihm verbleibende moralisch-ethische Denkweise. In seiner radikalen Selbstverfehlung verdrängt er zugleich menschlich-paradox sich selbst kleinmachend, die ungeheure Herabsetzung des Menschen, die nicht nur den technologischen Bereich betreffend „darin liegt, dass man gebrauchen müsse, was möglich sei“, denn ist es nicht so, dass „die halbe Ethik auf dem Neinsagenkönnen ruht" und ist es somit als Kulturschaffender und -vermittelnder „nicht wirklich unwürdig anzunehmen, dass die Erwachsenen kindischer seien als die Kinder?“ (Wenzl 1946, vgl. S. 28). Es ist ein stückweit amüsant zu beobachten, wie sich ohne eine notwendige, sich bescheidende, objektivierende Distanzierung von der Selbstbezogenheit ihr Mittelpunkt um sich selbst kreist und nichts gebiert. Immer nach dem gleichen groben Schema, wie z.B.: Flussläufe begradigen, dann renaturieren und Schäden begrenzen; genmanipulierte Nahrungsmittel essen und dann durch Biokostherstellung und deren Verköstigung Schäden begrenzen usw. in aeternum. Macht haben und Macht verlieren gehen hier Hand in Hand, und sind niemals für sich eine vereinzelte Totalität. Die Differenz von Anspruch und Wirklichkeit 
kann wirksam durch ein über sich und den Dingen stehendes Lachen ermittelt und gegebenfalls korrigiert werden. Zumindest ist die Ironie ein kleiner Anfang davon, dem Tod im Spiegel zu entrinnen, denn die „Ironie ist eben auch eine Form, den Tod ins Leben zu lassen, weil sie jene Distanz vom Leben einübt [...]. Und der Tod? Der ist die Negation der Zweckreihen, [...]“ (Reemtsma 2006, S. 423, 422). Nun betrifft aber eine Vision der Transhumanisten gerade die Überwindung des Todes, und könnte von daher auch als eine Position ,Jenseits aller Zwecke“ auftreten. Eine Position, die auf den ersten Blick einleuchtend erscheint, zumal nach der Abschaffung des Polytheismus und dem Ausruf Nietzsches, dass , Gott tot ist, weil wir ihn selbst getötet haben'. Seinem Ausruf könnten wir heutzutage getrost noch hinzufügen, das Heldentum ist tot', weil wir sie alle selbst getötet haben. Doch, das Sterben der Helden und der Heldenmythen trifft den Menschen direkt ins Mark, da er sich damit gleich selbst mitabschafft, und abstumpft. Man liebte die Helden und ,Antihelden', weil sie besser und empathischer waren und sind als die Durchschnittsmenschen.

Die Crux an diesem tarnenden Transhumanismus ist, dass er erst zu dem Zeitpunkt alle Zwecke abschaffen würde, wenn er einen Endzweck erreicht hat, d.h. alle Zwecke in diesem aufgegangen und kumuliert sind, und die Menschen darauf hin nivellierend geklont, modifiziert, geeicht und genormt sind. Ein Wiegen und zu leicht befinden ist bei solcher menschlichen Vermessung kaum mehr möglich. Doch selbst bei einer angenommenen möglichen Überwindung des Todes bestünde immer noch ein Hauch von Möglichkeit den transhumanistischen Endzweck als einen erlittenen Schmerz zu erahnen oder zu erfahren und mit Wieland festzustellen: „Aber wie groß diese Vergütung auch seyn möchte, kann sie machen, daß ich nicht gelitten habe, was ich leiden mußte? Ein Zug aus dem Lethe ist in solchen Fällen die beste Entschädigung" (Reemtsma 2006, S. 417). Lediglich ein nichts empfindender Mensch, ergo ein gänzlich abgeschaffter oder eine gewisse Art transhumanistischer Mensch, könnte solch einen Denkkomplex verhindern. Wenn nicht, wäre der transhumanistische Ansatz womöglich schnell in Frage gestellt. Die wahre Kunst einer Position des ,Jenseits aller Zwecke“ besteht hingegen darin, ein Jenseits aller Zwecke trotz einer gegebenen Vielfalt von Zwecken zu finden. Die Ironie und vielmehr noch das Lachen weisen den Weg. „Durch Lachen tödtet man“, wie Nietzsche in diesem Sinne so schön lakonisch sagt (Nietzsche, KSA 4, S. 392).

Jede einzelne Zweckbindung und jede einzelne Idee steht immer vereinzelt mit beschränkter Dachwirkung, da die Integration in sie immer nur über jedes einzelne Individuum funktioniert und nur bis zu einem bestimmten Grad über eine Gruppenzugehörigkeit oder Zwang zu erreichen ist. So wie die Gedanken frei sind, sind es die Gefühle selbstverständlich auch, wahrscheinlich sogar in einem viel stärker ausgeprägten Maße, da sie das Unbewusste miterfühlen können. Jeder Mensch trägt seine ureigene Wahrheit in sich und bewegt sich in seinem eigenen Lebenskontext und Umfeld, was von Ideologen aller Art zugleich und dergleichen mittransformiert werden müsste. Verschiebende Relationen und Wahrnehmungen innerhalb des jeweiligen Lebenskomplexes führen $\mathrm{zu}$ sich verschiebenden Wertungen und Sichtweisen, die samt und sonders mit wichtigen und nichtigen Sinngebungen belegt sind. Besonders augenfällig sind die Moral und Ethik sowie die Politik teleologisch ausgerichtet. Technische Artefakte und Naturwissenschaften können, aber müssen nicht teleologisch angelegt sein. Auch ästhetische Gebilde und Kunstwerke sind in irgendeiner Weise sinnstiftend, aber trotz allen Umwertungen, auch ihrer selbst, bleiben sie sich treu und widerstehen auf eine recht eigenwillige Art und Weise allen sie missbrauchenden gesellschaftspolitischen Ideologien, für die gemeinschaftlich inbegriffen in die Geistes- und Sozialwissenschaften gilt: „Im Unterschied zu Religionen und Philosophien behaupten sie nicht, anderes zu sein. Kunst lügt nicht" (Reemtsma 2006, S. 422). Was hier im tiefsten Kern widersteht ist das Individuum, das in der Kunst, im Schönen und Ästhetischen, und im ,göttlichen' Lachen vogelfrei und unabhängig, nicht nur sein kann, sondern ist. Frei von Zwecken und Sinnzuschreibungen, Zeit und Grund umwertend. Aus sich und der Welt austretend und sich zugleich findend, auch Neues erfindend, als Individuum. So ist im Anfang nicht der Sinn, vom Zweck gleich ganz zu 
schweigen. Die Tat im Anfang gilt nur dem faustischen Machtmenschen etwas. Und das Wort im Anfang, - was kann es nicht lügen. Hier, in diesem Kontext, ist nicht der Ort um der Bedeutungsvielfalt und Tiefe des Logos nachzugehen, der weit über das gesprochene Wort hinausreicht. Vielmehr soll zu der folgenden Aussage übergeleitet werden: Im Anfang war die Vertiefung. Eine Empathie, die aus der Überfülle und Freude heraus durch ein emphatisches Auseinanderstreben eine Vertiefung der Dinge erreicht. Gleichwohl können diese sich aufspaltend ins Gegenteil kehren, auch wiederkehren, sich anziehen oder abstoßen, wie auch sterben und verändert entstehen. Aller Anfang wäre ästhetisch gedacht. Gegenüber diesem möglichen gedanklichen Aspekt befindet sich ein aufs Geratewohl nach faustischer Art herumtastender Trial-and-Error-Tatendrang von transhumanistischen Positionen, mit dem nicht näher erklärten, aber dezidierten Wunsch nach einer in Kauf genommenen irreversiblen Verschmelzung der Dinge, von teils ewigem Bestand.

\section{Die offen abgeschlossene Lücke}

Die räumliche Gestaltung sowie die zeitliche berührt den Menschen ganz offensichtlich in seinem innersten Wesenskern, egal von welcher Warte aus er blickt. In Heinrich von Kleist und mit seiner Schrift Über das Marionettentheater findet sich hierfür ein weiteres wunderbares Beispiel nach der Schachnovelle, aber nun auf das Leibliche bezogen reflektierend, und das Setting des Technischen wiederum miteinbeziehend.

Kleist, der sich gleichermaßen der „Mathematik und Philosophie, - als den beiden Grundfesten alles Wissens“ verschrieb, brach sein Mathematikstudium ab und war enttäuscht von der Philosophie, da sie ihm beide kein festes Fundament und keinen Halt bieten konnten und versucht deshalb brückenbauend den Dissens zwischen Logik und Rhetorik aufzulösen, der ihn zeitlebens beschäftigte (Kleist 1993, S. 479). Er geht in dem Dialog der Leitfrage nach, ob ein Maschinist ein Tänzer sein müsse, oder wenigstens einen Begriff vom Schönen im Tanz haben müsse, um eine Marionette mit so viel Anmut und Grazie führen zu können, wie ein Tänzer sie beim Tanzen entfaltet, oder ob er nicht sogar durch ein technisches Körper-Tuning perfekter sein könne als dieser. Des Weiteren stellte sich die allgemeine Frage, warum bei einem Menschen die Fähigkeiten und Eigenschaften schwinden, hier die Schönheit bzw. die Grazie der Geste und des Körperausdrucks, sobald er sich ihrer in der narzisstischen Spiegelfalle oder dem Inszenierungsdruck zwischen Erkünstelung und Natürlichkeit unter dem Blick des Anderen gewahr wird. Und weitergehend, falls es unter diesen Umständen nicht gelingt diese Eigenschaften zu nutzen, reicht es dann nicht, wenn man sich einen dressierten Tanzbären als perfekten Trainingspartner, wenn auch nicht mehr in der Tanzkunst, so doch in der sich gegenseitig belauernden, fintierenden und tänzelnden Fechtkunst erschaffen hat.

Man kann bemerken, dass jedes Mal ein Wechsel in ein anderes Medium stattgefunden hat und dabei etwas anderes erreicht und geschaffen wurde, allerdings nicht aus Freude, wie zum Beispiel beim ursprünglichen Balletttanz. Wenn die Puppen des Maschinisten den Boden nur noch wie die Elfen streifen, statt ihn wie Menschen zum Ruhen zu nutzen, dann gleichen sie den Raumfahrern in ihren Astronautenanzügen, die sich auf der Mondoberfläche hüpfend vorwärts bewegen, weil das Medium sprich das Gravitationsfeld sich geändert hat. Die Perfektionierung oder ein Wechsel in einen anderen Bereich kann nur gelingen, wenn es von inniger, ausgelassener Freude begleitetet ist. Ein gelungenes Beispiel hierfür liefert Ludwig van Beethovens 9. Sinfonie mit der Ode An die Freude von Friedrich Schiller. In ihr heißt es ganz zu Recht: ,Freude heißt die starke Feder /in der ewigen Natur. Freude, Freude treibt die Räder/ in der großen Weltenuhr" (Schiller 1786, S. 3).

Was ergibt sich nun rund zweihundert Jahre später im Hinblick auf die Perfektionierung und Technisierung unserer Lebensumgebung? In der Automatisierungstechnik ist man zur eigenen 
Verwunderung ihrer Befürworter vielfach an Grenzen gestoßen, um nur eine zu benennen: den Verlust von handwerklichem und geistigem Wissen, das man für die Programmierung der Roboter zuallererst voraussetzen muss. So erklärte der Toyota Konzern, dass er „100 Arbeitsbereiche schaffen wolle, in denen Roboter durch Menschen ersetzt werden", weil das Wissen der Handwerksmeister, im Japanischen als ,Götter' bezeichnet, verloren gehe und damit eine ,wichtige Quelle der Produktentwicklung“, die sich nicht aus den Maschinen selbst heraus abdecken lässt: „wir müssen unsere manuellen Fähigkeiten verbessern und weiterentwickeln und uns dabei nicht von Maschinen abhängig machen“" (vgl. Granitza/ Hauer 2017, Abs. 2-3). So ist es nach meinem Dafürhalten durchaus ebenso möglich und naheliegend, dass etwa die gichtgeplagten Hände innovativere Impulse für die Maschinen- und Produktentwicklung liefern könnten als die vermeintlich perfekten und gesunden. Mit Hilfe von Neuro-Chip-Implantaten an Mensch-Maschine-Schnittstellen und Genmanipulationen werden sich mit Sicherheit auch veränderte Gefühle und Empfindungen einstellen sowie gezielt manipulieren lassen, wobei zerstörerische oder Hochstimmungen wohl leichter zu induzieren sein werden, als die echte, bereichernde und beglückende Freude. So mag beispielsweise eine Mondfahrt als technisches Wunderwerk zwar einen großen Eindruck hinterlassen, doch der Blick ins All ist von ergreifender und unsagbarer Schönheit, und nicht zu verwechseln mit dem Endlichkeitsgefühl, das sich nach dem Geschauten einstellen kann.

Das neu entstehende Bioinformationsrecht, das die Körperteile und -informationsbestandteile des Menschen neu verhandelt, wird noch zu bisher ungeahnten Entwicklungen führen. Ob die Ausstrahlung des Menschen oder etwas jetzt noch Unbekanntes ebenso darunterfällt, wird sich finden und sein mahnendes Beispiel in Wielands Der Prozess um des Esels Schatten finden. In jener abderischen Gesellschaft, in der „es nichts kostete, von einer Extremität zur anderen überzugehen" wurde im Streit um das Mietrecht auf den Schatten eines gemieteten Esels der Schatten des Esels zu einem eigenständigen Eselsschatten und selbst die ,demokratische Staatseinrichtung“ zum „Schattenwerk und politischen Gaukelspiel“ (Wieland, S. 132, 65). Die eingefahrene Situation wurde zufällig, durch den aufgebrachten Mob, aufgelöst, in dem man den völlig unschuldigen Esel „,die Zeche zahlen“ ließ und ihn ,,in tausend Stücke“ zerriss; das nachfolgende, erkennende ,unauslöschliche Gelächter“ glich „den Göttern im ersten Buche des Ilias“ (Wieland, S. 130). Der anwesende Demokrit, der Begründer des Atomismus und , lachende Philosoph", kann einen Interpretationsweg aufzeigen. Durch die Symbolik des dionysischen Opfertods durch Zerreißen wird ein Neuanfang und eine neue Perspektive auf die Sicht der Dinge ermöglicht, wie auch die sich ständig aufs Neue polarisierenden, ideengeschichtlichen Positionen in ihrer Bedeutung relativiert, denen es an Situationskomik wahrlich nicht mangelt. Ästhetisches Denken allgemein bricht starre und lineare Entwicklungen auf, durchbricht Erwartungshaltungen, bringt Widersprüchliches zu Tage und birgt in seiner immerwährenden Offenheit sui generis das Geheimnis- und Rätselvolle. Der Lachende und der Tanzende sind beide sich Entziehende und zugleich ein auffordernder Logos. Wer nicht mitmacht, versteht auch nicht; nichts von diesem epistemologischen Weg und seinen Umwertungen, und nichts von der Choreographie, die den Menschen in seiner Ganzheit betrifft und leitet. Das ästhetische Denken ist ein eigenständiger, wenn auch schwer fassbarer Denkweg, der noch viele, weitere Aspekte und Ariadnefäden auf seinen Wegen vorzuweisen hat, und wie alle ernsthaften Denkansätze so komplex und umfänglich ist, dass er gewichtige Berührungspunkte zu anderen Bereichen aufweist. Naheliegend ist, unter der spinozischen Betrachtungsweise sub specie aeternitatis, die Berührung mit den Religionen und dem Christentum, wo etwa der tänzelnde Christus ein bekanntes Motiv ist. Die Verbindung von Kunst und Technik ist eine komplementäre, da der eine Bereich dem anderen seinen 
Verfallszustand aufzuzeigen vermag, desgleichen seine Optimierungsmöglichkeiten. Eine Abkürzung des Weges führt deshalb höchst selten oder überhaupt nicht ans Ziel. Und noch viel weniger erfolgreich wird man sein, wenn man das mühselige Geschäft des Denkens und Handelns, sogar das Menschseins selbst, Fremden oder schleichend den Künstlichen Intelligenzen überlassen will, die alle ,nur das Beste für einen wollen'. Deshalb möchte ich zum Schluss meiner Betrachtungen einen bemerkenswerten Satz von Steve Jobs, dem Mitbegründer von Appel Inc. und der allseits bekannten Persönlichkeit aus der Computerindustrie anführen, den man gerne öfter vernehmen und beherzigt sehen würde: „I would trade all of my technology for an afternoon with Socrates.“ („Ich würde meine gesamte Technologie für einen Nachmittag mit Sokrates eintauschen."Von Verf.) (Jobs 2001, Abs. 3).

\section{Literaturverzeichnis}

Anders, Günter (1961): Die Antiquiertheit des Menschen. Über die Seele im Zeitalter der zweiten industriellen Revolution. Bd. 1. München: C. H. Beck.

Cebrian, Manuel/Rahwan, Iyad/Yanardag, Pinar (2018): „Norman. World's first psychopath AI.“

http://norman-ai.mit.edu/, besucht am 31.1.2019.

Grigat, Felix (2019): „Bredouille“. In: Forschung \& Lehre, Nr. 26, S. 5.

Goethe, Johann Wolfgang von (1998): „Maximen und Reflexionen“. In: Goethe Werke, Jubiläumsausgabe, Bd. 6. Friedmar Apel/Hendrik Birus et.al. (Hrsg.). Darmstadt: Wissenschaftliche Buchgesellschaft.

Granitza, Eckart/ Hauer, Thomas (2017): „Roboter als die Killer der Kreativität.“ Von: Stuttgarter Zeitung, Stuttgarter Nachrichten.

https://www.ideenwerkbw.de/roboter-kreativitaet/, besucht am 31.1.2019.

Gruber, Malte-Christian (2015): Bioinformationsrecht. Zur Persönlichkeitsentfaltung des Menschen in technisierter Verfassung. Tübingen: Mohr Siebeck.

Huxley, Aldous (2007): Schöne neue Welt. Frankfurt a.M.: Fischer.

Jobs, Steve (2001): „The Classroom Of The Future.“ Newsweek 28.10.2001

https://www.newsweek.com/classroom-future-154191, besucht am 31.1.2019.

Kleist, von Heinrich (1993): „Brief an Christian Ernst Martini am 18. März 1799“. In: Sämtliche Werke und Briefe, 9., vermehrte und revidierte Aufl., Bd. 2. Helmut Sembdner (Hrsg.). München: Hanser, S. 479.

Nietzsche, Friedrich: Werke. Kritische Gesamtausgabe. (KSA). In 15. Bde, 1967ff. Colli, Giorgio/Montinari, Mazzino (Hrsg.). München, Berlin: dtv/De Gruyter.

Ommeln, Miriam (2019): „Augmented Reality. Das Verschwinden der Farbe“. In: Oliver Ruf/Uta Schaffers (Hrsg.): Kleine Medien. Kulturwissenschaftliche Lektüren. Würzburg: Königshausen \& Neumann. 
Ommeln, Miriam (2017): Wissensgesellschaft quo vadis? Was heißt Wissen und zu welchem Ende soll es führen? Karlsruhe: KIT Scientific. DOI: 10.5445/IR/1000080060, besucht am 31.1.2019.

Ommeln, Miriam (2016a) Potenzialräume des Zeitbegriffs: Residuum und Desiderat der heutigen Technikphilosophie. Karlsruhe: KIT Scientific, Karlsruhe. DOI: 10.5445/IR/1000080491, besucht am 31.1.2019.

Ommeln, Miriam (2016b): Die Natur ist a-moralisch. Genormt durch ,Zirkel und Lineal 'ist sie als rechtes Maß für den Menschen unzureichend. Nietzsches Sicht auf den Naturbegriff. Karlsruhe: KIT Scientific. DOI: 10.5445/IR/1000080492, besucht am 31.1.2019.

Ommeln, Miriam (2014a): „Die ethische Janusköpfigkeit der Medienkunst: die Blickwinkel von Kunst und Informatik“. In: Matthias Maring (Hrsg.): Bereichsethiken im interdisziplinären Dialog. Karlsruhe: KIT Scientific, S. 403-418. DOI: 10.5445/IR/1000038379, besucht am 31.1.2019.

Ommeln, Miriam (2014b): „Ethik des Kopierens und die Philosophie des Transhumanismus“. In: (Hrsg.): Karsten Berr/Jürgen H. Franz: Prolegomena - Philosophie, Naturwissenschaft und Technik. Berlin: Frank \& Timme, S. 153-168. DOI: 10.5445/IR/1000080019, besucht am 31.1.2019.

Ommeln, Miriam (2010): On Upgrading Human Beings and Cyborgs in Posthumanism and the Question of Boredom. Karlsruhe: KIT Scientific. DOI: 10.5445/IR/1000080490, besucht am 31.1.2019.

Ortega y Gasset, José (1949): Betrachtungen über die Technik. Stuttgart: Deutsche VerlagsAnstalt.

Pais, Abraham (1986): „Raffiniert ist der Herrgott... “. Albert Einstein. Eine wissenschaftliche Biographie. Übersetzt von Roman U. Sexl et.al. Braunschweig, Wiesbaden: Vieweg.

Physikalisch-Technische Bundesanstalt (2017): Das neue Internationale Einheitensystem (SI). Braunschweig: Physikalisch-Technische Bundesanstalt (PTB).

Plessner, Helmuth (2003): Macht und menschliche Natur. Gesammelte Schriften V. Frankfurt am Main: Suhrkamp.

Rancière, Jacques (2008)): Ist Kunst widerständig? Übersetzt, um ein Gespräch mit Jacques Rancière und ein Nachwort erweitert von Frank Ruda/ Jan Völker (Hrsg.). Berlin: Merve.

Reemtsma, Jan Philipp (2006): „Leben heißt streben lernen“. In: Karl Hein Bohrer/ Kurt Scheel (Hrsg.): Merkur. Deutsche Zeitschrift für europäisches Denken. 60. Jahrgang, S. 410-424.

Schiller, Friedrich (1786): „An die Freude“. In: Thalia 1. Bd., Heft 2, S. 1-5.

The Future of Life Institute (FLI) (2015): „An Open Letter. Research Priorities for Robust and Beneficial Artificial Intelligence.“

https://futureoflife.org/ai-open-letter/, besucht am 31.1.2019. 
Tobben, Irene (2018): „Über frühe Versuche, durch Kunst die Welt zu retten. Homer und die Folgen“. In: Aida Bosch/ Hermann Pfütze (Hrsg.): Ästhetischer Widerstand gegen Zerstörung und Selbstzerstörung. Wiesbaden: Springer. S. 187-196.

Wenzl, Aloys (1946): Die Technik als philosophisches Problem. München: Pflaum.

Wieland, C.M. (o.J.): Der Prozess um des Esels Schatten. Mit Anmerkungen und einem Nachwort von Carlheinz Schellenberg (Hrsg.) Wiesbaden: Limes.

Zweig, Stefan (1979): Schachnovelle. Mit einem Nachwort von Siegfried Unseld. Frankfurt a. M.: Fischer. 